\title{
Human retinal photoreceptor cells in glaucoma: destructive changes of mithochondria and mitophagy
}

\author{
Natalia Obanina \\ Laboratory of ultrastructural research, \\ Research Institute of Clinical and \\ Experimental Lymphology - Branch of \\ the Institute of Cytology and Genetics, \\ Siberian Branch of the Russian \\ Academy of Sciences; \\ Section of cytology and genetics, \\ Department of Natural Sciences, \\ Novosibirsk State University \\ Novosibirsk, Russia \\ n.obanina@g.nsu.ru \\ Aleksandr Trunov \\ The academician S.N. Fyodorov \\ Federal State Institution "Intersectoral \\ Research and Technology Complex \\ "Eye Microsurgery", Ministry of \\ Healthcare of Russian Federation, \\ Novosibirsk Branch, \\ Novosibirsk, Russia \\ trunov1963@yandex.ru
}

\author{
Nataliya Bgatova \\ Laboratory of ultrastructural research, \\ Research Institute of Clinical and \\ Experimental Lymphology - Branch of \\ the Institute of Cytology and Genetics, \\ Siberian Branch of the Russian \\ Academy of Sciences \\ Novosibirsk, Russia \\ n_bgatova@ngs.ru
}

\author{
Valerii Chernykh \\ The academician S.N. Fyodorov \\ Federal State Institution "Intersectoral \\ Research and Technology Complex \\ "Eye Microsurgery", Ministry of \\ Healthcare of Russian Federation, \\ Novosibirsk Branch, \\ Novosibirsk, Russia \\ rimma@mntk.nsk.ru
}

\begin{abstract}
The leading place in the development of low vision and blindness worldwide is occupied by glaucoma. This is a disease of non-infectious nature, accompanied by an increase in intraocular pressure and degeneration of retinal neurons, optic nerve, as well as its spread in the central section of the visual analyzer. The aim of the study was to identify features of the ultrastructure of the human retinal photoreceptor cells in glaucoma. The study was carried out using transmission electron microscopy and morphometry. The volume densities $\left(V_{v}\right)$ of mitochondria and autophagosomes containing mitochondria were determined. Disorders of orientation and swelling of rod neurons membrane disks in the outer segments were detected. It is shown that rods are more exposed to destructive changes than cones.
\end{abstract}

Keywords - glaucoma, photoreceptors, ultrastructure, mitophagy

\section{INTRODUCTION}

Glaucoma is a multifactorial disease characterized by progressive damage and death of retinal ganglion cells, axon atrophy, and, as a result, optic neurodegeneration $[2,3]$. The process of neurodegeneration distributed outside of the retina and optic nerve to the central section of the visual analyzer $[2,4,5,6]$. It is known that glaucoma-associated cell death occurs by apoptosis. Apoptosis is caused by oxidative stress through mitochondrial damage, inflammation, endothelial dysregulation and dysfunction, and hypoxia [7]. Autophagy is a complexly organized process that is necessary for any eukaryotic cell for normal life. Dysfunction of autophagy at different stages in neurons leads to the emergence of many neurodegenerative diseases, in particular - glaucoma [8]. The pathogenesis of glaucoma is being studied on various animal models (mice, rats, rabbits, cats, monkeys, etc.). For example, it was shown that in the model of glaucoma, destructive changes in mitochondrial cristae develop in the rats retina. The swelling of the mitochondria is in the cytoplasm of neurons of the inner nuclear and inner plexiform layers (in ganglion cells), and photoreceptor pigment cells [9]. However, data obtained in animal models cannot be fully interpreted for the human eye due to anatomical and physiological differences. There are existing data about damage and ultrastructural changes in retinal ganglion cells, inner nuclear layer, inner plexiform layer $[3,10]$, but there is no information about ultrastructural changes in the photoreceptors in glaucoma.

\section{METHODS AND ALGORITHMS}

The study was conducted in accordance with the principles of the Helsinki Declaration of the World Medical Association "Ethical Principles for Scientific Medical Research with the Participation of Human", Federal Law of the Russian Federation of November 21, 2011 № 323 “About the Basics of Protecting the Health of Citizens in the Russian Federation", and the requirements Federal Law of July 27, 2006 № 152 (as amended on July 21, 2014) "About Personal Data" (as amended and supplemented, entered into force on September 1, 2015). The object of the study were retinal fragments of patients enucleated by medical indications of the eye with terminal stage of glaucoma of the Novosibirsk branch of Academician S.N. Fyodorov Federal State Institution «Intersectoral Research and Technology Complex «Eye Microsurgery», Ministry of Healthcare of Russian Federation. The study was conducted with the permission of the bioethical committee. The written informed consent of the patients to study the biological material was obtained. The collection of material was carried out by employees of the Novosibirsk branch of the IRTC «Eye Microsurgery».

Fragments of the human retina (about $1 \mathrm{~mm}^{3}$ ) were fixed in a $4 \%$ solution of paraformaldehyde prepared on Hanks medium, fixed for 1 hour in a $1 \%$ solution of $\mathrm{OsO}_{4}$ in phosphate buffer $(\mathrm{pH}=7.4)$, dehydrated in increasing concentration of ethanol, and then enclosed in Epon (Serva, Germany). Semi-thin sections with thick $1 \mu \mathrm{m}$ were prepared 
from the obtained blocks on a ultratome Leica UC7/FC7 (Germany / Switzerland), stained with toluidine blue. Then we studied under a light microscope "LEICA DME" and selected the necessary tissue sites for research in an electron microscope. Ultrathin sections thick 70-100 nm were obtained from the selected material, contrasted with a saturated aqueous solution of uranyl acetate and lead citrate, and studied using a electron microscope JEM 1400 (Japan). Microscopic analysis was carried out at the Multiple-access Center for Microscopy of Biological Subjects (Institute of Cytology and Genetics, Novosibirsk, Russia). Morphometry was performed using Image J software (Wayne Rasband, USA) with a closed test system at $\mathrm{x} 25 \mathrm{~K}$ magnification. The mean (M) and standard deviation (SD) were calculated using Microsoft Excel software. The significance of differences between the parameters was determined by Statistica 6.0 software (StatSoft, Inc.) with Mann-Whitney U-test. The differences were considered significant at $p<0.05$.

\section{RESULTS}

The volume densities $\left(\mathrm{V}_{\mathrm{V}}\right)$ of mitochondria and autophagosomes containing mitochondria in ellipsoids of both rod and cone cells inner segment were counted. In the rod cells outer segments swelling of membrane disks and their disorganization were noted. Disorder of the disk's structure contributes to the destruction of the transmembrane proteins of the visual cascade, which grab the photons. It reduces the photosensitive function of cells. In the rod ellipsoids turgescence of mitochondria was noted, the volume density of which was $4 \%$. Mitochondrial swelling was characterized by a multiple increase of area and a decrease in electron density of the mitochondrial matrix. Volume density of mitochondria with destructive changes of cristae was $20 \%$. Autophagosomes with mitochondria were observed (4.5\%). In the outer segments of cone cells the membrane semidisks were located more orderly. Mitochondrial swelling was not observed in their ellipsoids, single autophagosomes were noted $(0.6 \%)$. Cell death associated with glaucoma occurs through apoptosis [7]. It is initiated by many factors: a) relating to mitochondria directly (oxidative stress); b) affecting the development of apoptosis indirectly (Fasmediated signaling pathway, the effects of neurotoxic proteins: amyloid beta and tau protein) [7, 11]. During the study, apoptosis was not detected in the human photoreceptors. However, noted the autophagic activity may lead to the death of photoreceptors.

\section{CONCLUSION}

In our study mitochondria with destructive changes in cristae were detected both in rod and cone cells ellipsoids of all types of photoreceptors. In rods also present mitochondrial swelling and mitophagy. It was revealed that with glaucoma, rods are subject to greater disturbances in contrast to cones. It indicates their more pronounced reaction on an increase of intraocular pressure. Probably, autophagy can plays a dual role in retinal cells, on the one hand, contribute to the survival of cells by removing damaged structures, and on the other hand, initiate the apoptosis, which requires further research.

\section{ACKNOWLEDGMENT}

This work was supported with financing of the Novosibirsk Research Institute of Clinical and Experimental Lymphology as part of a state order, No. 0324-2019-0045.

\section{REFERENCES}

[1] V.V. Chernych, N.P. Bgatova, "Lymphatic structures of the human eye and their changes in pimary open-angle glaucoma," Ophthalmology, Moscow, 80 pp., 2019.

[2] V.P. Erichev, V.P. Tumanov, L.A. Panushkina, "Glaucoma and nuerodegererative diseases," Glaucoma, Vol. 1, pp. 62-68, 2012.

[3] M. Almasieh, A.M. Wilson, B. Morquette, J.L. Cueva Vargas and A. Di Polo, "The molecular basis of retinal ganglion cell death in glaucoma," Progress in Retinal and Eye Research, vol. 31(2), pp. 152181, 2012.

[4] J.A. Ghiso, I. Doudevski, R. Ritch, A.A. Rostagno, "Alzheimer's Disease and Glaucoma: Mechanistic Similarities and Differences," Journal of Glaucoma, Vol. 22, pp. 36-38, June-July 2013.

[5] F.G. Garaci, F. Bolacchi, A. Cerulli, M. Melis, A. Spanò, C. Cedrone, R. Floris, G. Simonetti, C. Nucci, "Optic Nerve and Optic Radiation Neurodegeneration in Patients with Glaucoma: In Vivo Analysis with 3-T Diffusion-Tensor MR Imaging," Radiol, Vol. 252 (2), pp. 496501, 2009.

[6] M.D. Pinazo-Duran, K. Shoaie-Nia, V. Zanon-Moreno, S.M. SanzGonzalez, J.B. Del Castillo, J.J. Garcia-Medina "Strategies to reduce oxidative stress in glaucoma patients," Curr Neuropharmacol, vol. 16(7), pp. 903-918, 2018.

[7] I.R. Gazizova, I.Yu. Tikhomirova, "The role of mitochondrial dysphunction in glaucoma," Medical bulletin of Bashkortostan, Vol. 10(2), pp. 153-156, 2015.

[8] I.A. Kochergin, M.N. Zakharova, "The Role of Autophagy in Neurodegenerative Diseases," Neurochemistry, vol. 33(1), pp. 12-24, 2016.

[9] H. Celicer, N. Yuksel, S. Solakoglu, L. Karabas, F. Aktar, Y. Caglar, "Neuroprotective Effects of Memantine in the Retina of Glaucomatous Rats: An Electron Microscopic Study," J Ophthalmic Vis Res., vol. 11(2), pp. 174-82, April-June 2016.

[10] T.C. Nag, and S. Wadhwa, "Ultrastructure of the human retina in aging and various pathological states," Micron, vol. 43(7), pp. 759-781, 2012.

[11] E.A. Egorov, V.N. Alekseev, I.R. Gazizova, E.B. Martynova, "Mitochondrial morphological changes of tracular cells in patients with primary open-angle glaucoma," Russian Journal of Clinical Ophthalmology, vol. 16(3), pp. 137-139, March 2016. 